\title{
Procesos de investigación en doctorados de artes y diseño, estudio de caso comparativo entre la Universidad de Caldas y la Universidad de São Paulo
}

\section{Resumen}

En este artículo se sintetiza un estudio sobre la investigación creación en tesis doctorales de artes y diseño. El objetivo principal de la indagación era examinar el estado de la investigación basada en prácticas creativas en doctorados de artes y diseño. Su alcance fue comprender la participación de la actividad creadora en seis tesis realizadas en dos contextos particulares de formación de investigadores. El método empleado consistió en un estudio de caso comparativo entre el programa de Doctorado en Diseño y Creación de la Universidad de Caldas en Colombia y el Doctorado en Artes Visuales de la Universidad de São Paulo en Brasil. Para esto se dilucidaron dos proposiciones teóricas sobre el aporte teórico al conocimiento y la fiabilidad metodológica de las tesis doctorales adelantadas con prácticas creativas. Los principales resultados muestran las tendencias de cada caso, presentadas como códigos clasificados de acuerdo a dos categorías coherentes con las proposiciones del estudio. Frente a la categoría conocimiento, la comparación de los casos presenta concordancias entre los códigos procesos, práctica y creación. Los códigos de la categoría metodología demuestran coincidencias entre métodos diversos, práctica creativa, narración, cualitativa y registro audiovisual. Se concluye que la investigación creación puede generar conocimiento útil a diversas disciplinas, por lo que debe ser expuesta en un lenguaje académico común y explícito, favoreciendo la trasmisión, el debate, la negación o la afirmación de las teorías resultantes. También se confirman las teorías respecto a la diversidad metodológica y el aprovechamiento de la creación para la recolección y procesamiento de datos. Se destaca la conveniencia de evidenciar el plan de investigación, develando plenamente las prácticas creativas y su articulación con la construcción teórica.

\author{
Antonio Stalin García Ríos \\ Doctor en Diseño y Creación \\ Universidad de Pamplona, \\ Pamplona, Colombia. \\ Correo electrónico: \\ antoniostalin@unipamplona.edu.co \\ ๑ orcid.org/0000-0002-1788-8478 \\ Google Scholar
}

Recibido: julio 01 de 2018 Aprobado: abril 14 de 2020

Palabras clave:

Artes, Creación, Diseño, Investigación, Tesis. 


\section{Research processes in arts and design in doctoral programs, a comparative case study between Universidad de Caldas and Unversidade de São Paulo}

\begin{abstract}
This article synthesizes a study on research-creation in doctoral theses of arts and design. The main objective of the inquiry was to examine state of research based on creative practices in doctoral programs of arts and design. Its scope was to understand the participation of creative activity in six theses carried out in two specific research training contexts. The method used consisted of a comparative case study between the doctoral program in design and creation of Universidad de Caldas, Colombia and the doctoral degree in visual arts of Universidade de São Paulo, Brazil. For this purpose, two theoretical propositions were elucidated on the theoretical contribution to knowledge and the methodological reliability of the doctoral theses carried out with creative practices. The main results show the trends in each case, presented as codes classified according to two categories consistent with the proposals of the study. Compared to the knowledge category, the comparison of the cases shows concordance between the codes of processes, practice and creation. The codes of the methodology category show similarities between diverse methods, creative practice, narrative, qualitative and audiovisual recording. It is concluded that research-creation can generate useful knowledge to various disciplines, so it must be exposed in a common and explicit academic language, favoring the transmission, debate, negation or affirmation of resulting theories. Theories regarding methodological diversity and the use of creation for the collection and processing of data are also confirmed. The convenience of highlighting the research plan is emphasized fully revealing the creative practices and their articulation with theoretical construction.
\end{abstract}

Key words:

Arts, Creation, Design,

Dissertation, Research. 


\section{Introducción}

El debate sobre la investigación doctoral basada en prácticas creativas surge porque la formación de investigadores se realiza en el marco de los programas doctorales, como nivel educativo superior. En un doctorado se prepara personal idóneo para la investigación original, con resultados útiles para el crecimiento de un campo del conocimiento (Friedman, 2014). Por supuesto, los programas doctorales de artes y diseño no pueden ser ajenos a este propósito formativo. Sin embargo, existe un debate internacional sobre los proyectos de investigación basados en la práctica creativa y la necesidad de formar doctores con competencias creadoras o investigadoras (Friedman \& Ox, 2017). Con la discusión se procura alcanzar garantías de una metodología válida, productora de conocimiento teórico aprovechable (Cross, 1993). Esta confrontación académica implica comparaciones entre la investigación artística y la investigación en sí, y sobre los diferentes tipos de conocimiento posibles (Borgdorff, 2010). Como consecuencia de los desacuerdos, se afecta la evaluación ecuánime entre pares académicos respecto de los resultados obtenidos, lo cual impide demostrar la relevancia del conocimiento alcanzado y obstaculiza el aprovechamiento e impacto de una disertación a nivel de doctorado.

No obstante, importantes autores de investigación académica en arte y diseño permiten inferir que la investigación doctoral basada en prácticas creativas es factible, siempre y cuando se pueda verificar el cumplimiento de los criterios exigidos en cualquier investigación propiamente dicha (Archer, 1995; Belo, 2010; Durling \& Niedderer, 2007; Sullivan, 2011). Los criterios que refieren estos autores provienen de la investigación científica: sistematización, rigor metodológico, suficiencia teórica y aporte al conocimiento transmisible y evaluable. Un recurso necesario para evidenciar esta vía investigativa es el 
registro planeado, detallado y permanente sobre el proceso creativo realizado (Belo, 2010; Durling \& Niedderer, 2007).

La problematización incluye cuestionamientos como ¿La investigación que se adelanta a través de la práctica creativa, es válida como tesis doctoral? ¿La preparación requerida en la formación doctoral debe tener fines creadores, investigadores o pedagógicos? ¿Es posible difundir y discutir el conocimiento logrado a través de las obras creadas? ¿Cuáles son los tipos de conocimientos alcanzables a través de la investigación creación? ¿Cómo garantizar la fiabilidad de los métodos de investigación basados en prácticas creativas? La formulación del problema se centra en comprender ¿Cómo son los procesos de investigación a través de prácticas creativas en doctorados de artes y diseño?

\section{Método}

Para describir las condiciones en las que se desarrollan los procesos de investigación-creación en el sistema universitario de alto nivel en Colombia se procura un contraste internacional. Este objeto de estudio en emergencia es complejo y poco estudiado en Colombia, por lo que es necesario analizarlo contextualmente mediante un enfoque de investigación comprensivo. El método, en concordancia con la cosmovisión del autor, es un estudio de caso comparativo en el que se prioriza el contraste de los contextos, analizando las semejanzas, diferencias y patrones de dos o más casos con fines comunes, para lo que se requiere comprender y caracterizar las especificidades de cada caso a profundidad (Goodrick, 2014). Esta vía exige la selección de un caso de referencia que permita llegar a conclusiones generales respecto al fenómeno estudiado. De esta manera, se lleva a cabo "Una investigación empírica que estudia un fenómeno contemporáneo dentro de un contexto de vida real, especialmente cuando las fronteras entre fenómeno y contexto no son claramente evidentes" (Yin, 1994, p. 13). 
Para la selección de los casos se realizó un inventario donde se identificaron 66 programas doctorales en artes y diseño en Latinoamérica. El primer caso corresponde al programa de formación posgradual donde se defendieron las tres primeras tesis doctorales que asumieron la investigación creación en Colombia. El segundo caso se elige al encontrar que el $62,12 \%$ de esos doctorados se adelantan en Brasil, identificando el programa de formación doctoral con mayor trayectoria investigativa en las artes de Latinoamérica. Así, los casos comparados son el doctorado en Diseño y Creación de la Universidad de Caldas en Colombia y el doctorado en Artes Visuales de la Universidad de São Paulo en Brasil. Una vez seleccionados los casos, el diseño de investigación exige la definición de cinco aspectos fundamentales (Yin, 2003), los cuales se desarrollan así:

Pregunta. ¿Cómo es la investigación a través de prácticas creativas en los doctorados que se comparan?

Proposiciones. A partir de la revisión teórica adelantada se plantean dos proposiciones a verificar: 1) La I+C aporta teoría al conocimiento. 2) La I+C es metodológicamente confiable.

Unidades de análisis. Para cada caso se analizan tres tesis doctorales con investigaciones basadas en prácticas creativas. Para el caso colombiano, las unidades de análisis que se incrustan corresponden a las tres primeras tesis doctorales en las que sus respectivos autores declararon el uso de la investigación-creación. Estas tres tesis fueron defendidas exitosamente en el programa de doctorado en diseño y creación de la Universidad de Caldas y son:

- Tesis de Roberto Cuervo Pulido: ¿Cómo escuchar la ciudad? La experiencia de los paisajes sonoros urbanos (2016). 
- Tesis de Sandra Johana Silva Cañaveral: Vestidos Orales y la visibilización de las identidades transgénero en el arte. Una reflexión en torno a la relación entre creación e investigación (2016).

- Tesis de Daniel Enrique Ariza: Huellas digitales. Performance digital como profanación del dispositivo prisión (2016).

En el caso brasileño la participación de la práctica creativa en las tesis doctorales es aceptada en dos líneas de investigación del doctorado en artes visuales de la Universidad de São Paulo, las tres unidades de análisis incrustadas son tesis con temas y años diferentes:

- Tesis de Ricardo Roclaw Basbaum: ¿Você gostaria de participar de uma experiência artística? (+ NBP) (2008).

- Tesis de Clarissa Ribeiro Pereira de Almeida: Instantes de metamorfose: o coletivo como processo; o processo como sistema (2011).

- Tesis de Daniel Peixoto Ferreira: Expressividade procedural: narrativas e jogos digitais (2016).

En total son seis unidades de análisis en dos contextos universitarios distintos.

Lógica entre los datos y las proposiciones. Para definir la coherencia entre los datos y las proposiciones primero se respondió a la pregunta ¿Qué datos se deben buscar para lograr la información que permita discutir las proposiciones teóricas formuladas? La categorización apriorística vincula cada proposición a una categoría y a los aspectos que las desarrollan en una investigación. Con esto, los datos de la categoría conocimiento se busca en los objetivos, hipótesis, resultados y conclusiones. Los datos de la categoría metodología se buscan en el apartado correspondiente de la tesis, planeación, método, técnicas e instrumentos y registro. 
Criterios para interpretar los resultados. Después de recaudar los datos, se agrupa lo hallado entre las tres unidades de análisis de cada caso, luego se clasifican los datos bajo criterios de frecuencias y patrones. Esto permite organizar los resultados en una matriz de categorías de acuerdo a las coincidencias y diferencias entre cada uno de los casos para determinar las tendencias respecto a cada proposición.

Instrumentos de recolección de información. Este recaudo de datos se consolida mediante fuentes como la búsqueda bibliográfica en bibliotecas universitarias de tesis doctorales, explorando la información publicada en internet como rankings, portales de las universidades y programas y normativas institucionales. Se aplica el análisis documental, la observación participante y diálogos con miembros de las comunidades académicas en estudio, in situ. Además, se utilizaron protocolos para la caracterización de los casos y para el análisis de las tesis, lo cual fue consignado en fichas de registro.

\section{Resultados}

Al analizar las tesis se descubrieron las principales tendencias de cada caso. Al comparar las semejanzas y diferencias de esas tendencias en los casos emergen los códigos para la interpretación final de las proposiciones teóricas, como se describe a continuación.

Diferencias entre las tendencias de las tesis frente a la categoría conocimiento:

- DDCUC' ${ }^{1}$ Énfasis en demostrar la potencialidad de la investigación creación como medio para acceder al conocimiento. Los códigos particulares son: Método y Comprensión. 
- DAVUSP2: Enfoque en los aportes teóricos alcanzables y creación con tecnología digital. Los códigos particulares son: Teoría, Tecnología digital y Enlace teórico-práctico.

Con las semejanzas de tendencias entre ambos casos se encuentran los códigos comunes: Procesos, Práctica y Creación. Estos patrones aportan argumentos para comprender la proposición 1 (conocimiento).

Al develar las tendencias de las unidades analizadas y comparando los casos respectivos, se descubre en ambos casos que la búsqueda de conocimientos explora la manera en que se desarrollan los procesos creativos. Las principales diferencias radican en la búsqueda del conocimiento desde las tesis doctorales. En el caso DAVUSP el foco es la teorización entrelazada con la práctica creativa. Por su parte, en el DDCUC se interesa en demostrar como la práctica creativa constituye una vía válida para la búsqueda del conocimiento. Esta particularidad se justifica en cuanto las tres unidades de análisis del caso colombiano fueron las tres primeras tesis doctorales defendidas como investigación creación en Nuestro País.

Para obtener los patrones o tendencias útiles para la comprensión de la proposición 2 se utilizó el mismo procedimiento. Diferencias entre las tendencias de las tesis frente a la categoría metodología:

DDCUC: La creación es el instrumento para la recolección de datos. Los códigos particulares son: Planes cambiantes y Creación colecta datos.

DAVUSP: Predominio de interacciones con obra y entre creadores. Los códigos particulares son: Interacciones y Experiencias. 
Con las semejanzas de tendencias entre ambos casos se encuentran los códigos comunes: Métodos diversos, Práctica creativa, Narración, Cualitativa y Registro audiovisual. Estos patrones aportan argumentos para comprender la proposición 2 (metodología).

Al comparar las tendencias que buscan respuestas para la proposición 2, entre las tesis de ambos doctorados existen significativas semejanzas en el abordaje metodológico. No presentan detalles de algún plan a seguir en el proyecto, continuando con sus propias experiencias y azares. Sin embargo, en ambos casos se descubren posturas cualitativas de las ciencias sociales y declaran la práctica creativa como método de investigación, en las que se destacan las narraciones propias o de terceros. Esto refleja y reafirma la diversidad de métodos reseñados en el marco teórico de la investigación. Otro consistente instrumento para el registro de los datos y el proceso son las grabaciones de audio y video. Se destaca la difusión en internet de las experiencias e interacciones con las obras en el caso DAVUSP. En ese programa la interacción constituye a la vez un propósito y una estrategia de investigación constante. Como se ha explicado, en Brasil la práctica creativa ha jugado un rol central en la investigación por lo que es usual la presentación en dos volúmenes (teórico y práctico) de la tesis final.

Con los códigos definidos se posibilita la respuesta de las proposiciones teóricas 1 y 2 . A continuación se condensan los resultados obtenidos con los datos recabados categorizados, codificados e interpretados.

Proposición 1: La investigación creación aporta teoría al conocimiento. La novedad en todas las tesis analizadas radica en la singularidad de los estudios y principalmente en sus desplazamientos hacia las fronteras de otros campos del conocimiento. La interdisciplinariedad y la transdisciplinariedad otorgan originalidad. Esto a la vez implica una exigencia en la trasmisión 
comprensible a las distintas áreas participantes. Al interpretar los códigos comunes de la categoría conocimiento se infiere que con la investigación creación se pueden emprender desarrollos teóricos, también comprender, explicar y mejorar los procesos de creación sobre todo cuando se recurre a la tecnología digital. Toda vez que la postura epistemológica implica una exploración directa del investigador, se destacan las búsquedas de los entrelazamientos teórico-prácticos de los proyectos. En este sentido, el otro propósito inherente a esta vía es la creación misma como producto.

Desde las tesis revisadas, hay una amplia gama de sectores que pueden beneficiarse de los resultados. Por supuesto, la exploración de los procesos y métodos de trabajo aporta principalmente a las áreas creativas. Pero las demás reflexiones que se suscitan dentro de las disertaciones derivan contribuciones aprovechables en campos como la acústica ambiental, la sociología, la justicia restaurativa, la industria cultural y de entretenimiento, los softwares o aplicaciones digitales, las nuevas tecnologías, la crítica, la filosofía, el diseño y las artes en general. Actividades que aumentan sus presupuestos y comprensiones de fenómenos que les impactan. Por su puesto, estos no son los únicos campos que se nutren de proyectos con creación, otras investigaciones mostrarán aportes en cualquier área o campo del conocimiento. Además, se evidencia el interés por legitimar desde la práctica, la investigación creación como método para la investigación en sentido estricto, este aspecto se valora de manera más pertinente en la proposición metodológica.

Respecto a la trasmisión del conocimiento, este debe ser claro y comprensible. Esta condición adquiere realce ante el escenario transdisciplinar de la investigación contemporánea, los campos intervinientes deben poder acceder a los conocimientos de forma plena y fluida. Por lo que los registros e informes deben ser interpretados y trascritos en un lenguaje explícito y común que garantice la lectura académica correspondiente al nivel doctoral 
y con esto se favorezca el acceso concreto a los hallazgos, resultados y conclusiones de la tesis.

Proposición 2: La investigación creación es metodológicamente confiable. La interpretación de los códigos comunes en la categoría metodología lleva a inferir que la investigación creación todavía adolece de la concepción de planes para ejecutar el proyecto. Este vacío es atribuido a cierta actitud creativa, aunque la práctica artística realmente es dirigida por guías individuales que pueden ser develadas (Gray \& Malins, 1993). En efecto, es posible explicitar la manera como se incorporan y registran sucesos como la continuación de proyectos o experiencias anteriores, los azares, las sorpresas, el recorrido por otras disciplinas, encuentros, etcétera. No obstante, el abordaje de los métodos es consistente entre los casos.

Aunque son usuales los métodos cualitativos traídos de las ciencias sociales (estudios de caso, entrevistas, encuestas, foros, etc.), predomina la diversidad de métodos. La práctica creativa (o estudios prácticos) se perfila como la principal vía de indagación, incluso como instrumento de colección y procesamiento de datos. Se destaca indefectiblemente la narración del proceso, acompañado por relatos propios y ajenos sobre las experiencias individuales o grupales con la práctica creativa. Estos métodos diversos de investigación enfatizan la experiencia y las interacciones, ya sea del mismo candidato, de los espectadores (co-creadores - artistas, probadores o usuarios) con la creación, o entre los participantes de un colectivo artístico. La reflexión y el análisis resultantes de la práctica creativa también pueden explicitarse.

El principal instrumento de registro son las fotografías, los portales de internet, las grabaciones de audio y video. Así se registran las narraciones, presentaciones y acontecimientos del proyecto, por supuesto, éstos quedan registrados en los mismos soportes tecnológicos. Se destaca el carácter minucioso y longitudinal 
del registro y su disposición en los anexos. Sin embargo, en virtud de la transparencia y acceso a otras disciplinas, es indispensable generalizar la práctica de transcribir, interpretar y analizar dichos registros para la construcción teórica de la tesis.

En la discusión de resultados se confirma que con la investigación creación se genera conocimiento útil a diversas disciplinas, por lo que debe ser presentado en un lenguaje académico común y explícito. Para favorecer la trasmisión, el debate, la negación o la afirmación del conocimiento, estas disertaciones exponen las teorías resultantes de la investigación en un texto escrito serio y completo (Belo, 2010; Durling \& Niedderer, 2007; Eco, 1988; McNiff, 2008; Yee, 2010). En la complejidad del mundo actual, una comunicabilidad clara potencia la transdisciplinariedad y el crecimiento conjunto de los métodos creativos de investigación para acceder a conocimientos no alcanzables con enfoques convencionales. Una forma de lograr este propósito es el cumplimiento cabal de las normas establecidas en las instituciones para la presentación de una narrativa (Friedman, 2014) con los componentes, secciones o ítems mínimos del informe escrito final. Así mismo resulta conveniente la opción de presentar la tesis de investigación creación en dos volúmenes, uno para la teoría (investigación) y otro para la práctica (creación).

Esta comparación también concluye que los procedimientos o métodos de investigación creación son confiables, porque se confirman empíricamente los supuestos teóricos estudiados respecto a su diversidad metodológica y el aprovechamiento de la creación para la recolección y procesamiento de datos (Belo, 2010; Durling \& Niedderer, 2007; Evans, 2010; Gray \& Malins, 1993; Leavy, 2015; Reilly, 2002). Para afianzar esta vía, conviene evidenciar un plan o diseño metodológico que devele las formas en que el creador adelanta su práctica creativa y la manera como esto se articula con el desarrollo teórico de la investigación (enlace teórico-práctico). Así mismo, los datos registrados en 
cualquier medio deben ser transcritos para su interpretación, análisis y difusión entre las disciplinas comprometidas.

\section{Conclusiones}

Entre las reflexiones finales de la investigación se enjuicia el discurrir de los procesos creativos por los campos de la academia científica universitaria. Un doctorado, como programa de posgrado, responde a los estándares académicos de la educación superior en los que han predominado los conocimientos positivistas desde la ciencia, las disciplinas, la generalización de resultados, la objetivación de los estudios, entre otros. No obstante, los conocimientos en las artes son diversos y a ellos se accede con propósitos y medios distintos, por supuesto, los sistemas de evaluación también difieren. Esta dicotomía lleva a reconocer el absurdo de adelantar procesos creativos bajo criterios científicos; como todas las disciplinas no son idénticas, no existe coherencia directa entre las actividades para avanzar en los conocimientos científicos y las comprensiones artísticas de manera paralela. Evidentemente sí hay unas metodologías artísticas, pero éstas no pueden parangonarse con los métodos de investigación científica, por lo que resulta complejo adelantar procesos creativos en los entornos investigativos de tradición científica en las academias universitarias superiores.

Por otra parte, al discernir las dos proposiciones del estudio de caso comparado mediante las categorías establecidas, las tendencias encontradas y los códigos definidos, se puede argumentar la comprensión particular sobre cómo son los procesos de investigación a través de prácticas creativas en doctorados de artes y diseño. Se confirmaron empíricamente los supuestos teóricos revisados sobre la diversidad metodológica de la investigación basada en la práctica y el aprovechamiento de la creación para la recolección y procesamiento de datos. En las tesis con creación, además de la práctica, se acude a las 
entrevistas, encuestas, historias de vida, panel de expertos, observación participante, talleres, diario de campo y otras técnicas e instrumentos de métodos provenientes de las ciencias sociales.

También se determinó la necesidad de afianzar este esfuerzo con un plan o diseño metodológico que devele las formas en que el creador adelanta su práctica y la manera como esto se articula con el desarrollo teórico de la investigación en el enlace teórico-práctico. Los datos registrados en cualquier medio deben ser transcritos y clasificados para su interpretación, análisis y difusión entre las disciplinas interesadas.

Las principales semejanzas entre los doctorados comparados se encuentran en las metodologías cualitativas de las ciencias sociales que predominan. La narración y el registro con audiovisuales son comunes y la diversidad metodológica se enriquece con el uso de la práctica creativa como medio para adelantar o complementar la pesquisa. Hace falta generalizar la práctica de identificar para el público interesado los planes de trabajo y los aspectos generales del proyecto, que ocasionalmente se obvian en el documento final.

Las diferencias más relevantes están en las búsquedas, mientras el caso colombiano procura demostrar la viabilidad de la creación como investigación y comprender los procesos creativos, el caso brasileño se centra en el enlace teórico práctico, la tecnología digital, las experiencias y las interacciones, siempre con foco en la teorización.

Finalmente, con el propósito de encontrar salidas hacia una articulación de las metodologías de las ciencias con los procedimientos de las artes, se propone un método que ayude difuminar límites, soslayar y menguar contradicciones entre ambas formas de conocer o comprender el mundo desde miradas diversas. Con lo anterior, y al examinar el estado de la investigación basada en 
prácticas creativas en doctorados de artes y diseño, se concibe esta definición: La investigación creación es un método cualitativo útil para ampliar los conocimientos sobre las potencialidades de los materiales, los procesos, los conceptos y las funcionalidades de las obras, acciones o creaciones en artes y diseño. Estas posibilidades se nutren desde, y aportan a otras disciplinas, por lo que se transmiten con lenguajes comprensibles en la academia. Su principal estrategia es la experiencia reflexivo-creativa que entrelaza la teoría y la práctica.

\section{Referencias bibliográficas}

Almeida, C. R. (2011). Instantes de metamorfose: o coletivo como processo; o processo como sistema [Tesis Doctoral, Universidad de São Paulo]. https://repositorio.usp.br/item/002188690

Archer, B. (1995). The Nature of Research. Co-design. Interdisciplinary Journal of Design, 2(11), 6-13.

Ariza, D. E. (2016). Huellas digitales. Performance digital como profanación del dispositivo prisión [Tesis doctoral, Universidad de Caldas].

Basbaum, R. R. (2008). Você gostaria de participar de uma experiência artística? (+ NBP) [Tesis Doctoral, Universidad de São Paulo]. https://repositorio.usp. br/item/001728936

Belo, M. (2010). Doctoral Research in Art and Design. lade, Unidcom I, Av D Carlos, 1-7.

Borgdorff, H. (2010). El debate sobre la investigación en las artes. Cairon. Revista de estudios de danza (13), 25-46.

Cross, N. (1993). Editorial. Design Studies, 14(3), 226-227. 
Cuervo, R. (2016). ¿Cómo escuchar la ciudad? La experiencia de los paisajes sonoros urbanos. Manizales [Tesis doctoral, Universidad de Caldas]. http://biblio. ucaldas.edu.co:8000/cgi-bin/koha/opac-detail.pl?biblionumber=63865

Durling, D., \& Niedderer, K. (2007). The Benefits and limits of investigative designing. In Proceedings IASDR07. Hong Kong. https://www.sd.polyu. edu.hk/iasdr/proceeding/papers/The\%20Benefits\%20and\%20Limits\%20 of $\% 20$ Investigative $\% 20$ Designing\%20\%20\%20.pdf

Eco, U. (1988). Cómo se hace una tesis: técnica y procedimientos de estudio, investigación y escritura. Bogotá: FICA-Fundación para la Investigacion y la Cultura.

Evans, M. (2010). Researcher practice: Embedding creative practice within doctoral research in industrial design. Journal of Research Practice, 6(2), Article M16.

Ferreira, D. P. (2016). Expressividade procedural: narrativas e jogos digitais. [Tesis Doctoral, Universidad de São Paulo]. https://repositorio.usp.br/item/002780991

Friedman, K. (2014a). Now that we're different, what's still the same? En L. Ravelli, B. Paltridge, \& S. Starfield, Doctoral writing in the creative and performing arts (pp. 237-262). Faringdon: Libri Publishing.

Friedman, K. (2014b). Re: How "Design Thinking Research" and "Design Thinking" are related (or not)? Email discussion lists for the UK Education and Research communities. https://www.jiscmail.ac.uk/cgi-bin/ webadmin?A2=PHD-DESIGN;7c9e3890.1408

Friedman, K., \& Ox, J. (2017). PhD in Art and Design. Leonardo, 50(5), 515-519. DOI: 10.1162/LEON_e_01472

Goodrick, D. (2014). Estudios de caso comparativos, Síntesis metodológicas: evaluación de impacto $n .^{\circ}$ 9. Florencia: Centro de Investigaciones de UNICEF. 
Gray, C., \& Malins, J. (1993). Research procedures/methodology for artists \& designers. Aberdeen: Robert Gordon University. https://openair.rgu.ac.uk/ handle/10059/640

Leavy, P. (2015). Method meets art: Arts-based research practice. New York: Guilford Publications.

McNiff, S. (2008). Art-Based Research. En G. Knowles \& A. Cole, Handbook of the arts in qualitative research: Perspectives, methodologies, examples, and issues (pp. 29-40). Thousand Oaks, CA: SAGE.

Reilly, L. (2002). An alternative model of "knowledge" for the arts. Working Papers in Art and Design 2.

Silva-Cañaveral, S. (2016). Vestidos Orales y la visibilización de las identidades transgénero en el arte. Una reflexión en torno a la relación entre creación e investigación [Tesis doctoral, Universidad de Caldas]. http://biblio.ucaldas. edu.co:8000/cgi-bin/koha/opac-detail.pl?biblionumber=63586

Sullivan, G. (2011). The Artist as Researcher. New Roles for New Realities. En J. Wesseling, See it Again, Say it Again: The Artist as Researcher (pp. 79-101). Amsterdam: Valiz Antennal.

Yee, J. (2010). Methodological innovation in practice-based design doctorates. Journal of Research Practice, 6(2), Article M15.

Yin, R. (1994). Case Study Research. Design and Methods. London: SAGE.

Yin, R. (2003). Case Study Research. Design and Methods. USA: SAGE.

Como citar: García, A. S. (2020). Procesos de investigación en doctorados de artes y diseño, estudio de caso comparativo entre la Universidad de Caldas y la Universidad de São Paulo. Revista KEPES, 17(22), 59-75. https://doi.org/10.17151/kepes.2020.17.22.3 